\title{
The mediating role of cytokine IL-6 on the relationship of FEV, upon 6-minute walk distance in chronic obstructive pulmonary disease
}

This article was published in the following Dove Press journal:

International Journal of COPD

7 October 2014

Number of times this article has been viewed

\author{
Simonetta Baldi' \\ Paul E Jose 2 \\ Claudio Bruschi ${ }^{1}$ \\ Gian Domenico Pinna ${ }^{3}$ \\ Roberto Maestri ${ }^{3}$ \\ Antonella Rezzani ${ }^{1}$ \\ Ezio Bellinzona' \\ Claudio Fracchia' \\ Elena Dacosto' \\ Paola Crottil \\ Silvia Montemartini ${ }^{\prime}$ \\ 'Department of Pneumology, \\ Scientific Institute of Montescano, \\ Salvatore Maugeri Foundation IRCCS, \\ Montescano, Pavia, Italy; ${ }^{2}$ School \\ of Psychology, Victoria University, \\ Wellington, New Zealand; \\ ${ }^{3}$ Department of Biomedical \\ Engineering, Scientific Institute of \\ Montescano, Salvatore Maugeri \\ Foundation IRCCS, Montescano, \\ Pavia, Italy
}

Objectives: To explore the mediating role of protein interleukin-6 (IL-6) on the relationship between forced expiratory volume in 1 second $\left(\mathrm{FEV}_{1}\right)$ and 6-minute walk distance (6MWD) and, further, to determine whether status variables (such as age, sex, and body mass index [BMI]) operate as moderators of this mediation relationship.

Design: Moderated mediation model.

Setting: An inpatient pulmonary rehabilitation center in Italy.

Participants: All 153 patients involved in the screening of a randomized controlled clinical trial (ClinicalTrials.gov identifier: NCT01253941) were included in this study. All patients were Global initiative for chronic Obstructive Lung Disease (GOLD) stages I-IV and were aged $70.1 \pm 9.1$ years

Measurements: At run-in phase of the protocol, clinical and functional screening included BMI, fasting plasma levels of protein (IL-6), spirometry, and standardized 6-minute walking test, measured at the start of the respiratory rehabilitation program.

Methods: The size of the indirect effect of the initial variable $\left(\mathrm{FEV}_{1}\right)$ upon the outcome variable (6MWD) through the intervening variable (IL-6) was computed and tested for statistical significance. Moderated mediation analyses were subsequently conducted with age, sex, and BMI.

Results: $\mathrm{FEV}_{1}$ averaged $53.4 \% \pm 21.2 \%$, and $6 \mathrm{MWD} 66.4 \% \pm 41.3 \%$ of predicted. Median protein IL-6 was $6.68 \mathrm{pg} / \mathrm{mL}$ (interquartile range: 5.96). A bootstrapped mediation test supported the predicted indirect pathway $(P=0.003)$. The indirect effect through IL-6 log units accounted for $17 \%$ of the total effect between FEV and 6MWD. Age functioned as a significant moderator of the mediational pattern. For individuals aged $<70$ years, the standardized indirect effect was significant $(0.122,95 \%$ confidence interval $[\mathrm{CI}]: 0.044-0.254, P=0.004)$, and for individuals $>70$ years it was not significant $(0.04,95 \% \mathrm{CI}$ : -0.010 to $0.142, P=0.10)$.

Conclusion: This moderated mediation result based on concurrent data suggests, but does not prove, a causal role of systemic inflammatory syndrome on progression from functional impairment to "frailty" status and substantial disability in aging chronic obstructive pulmonary disease.

Keywords: COPD, chronic airflow obstruction, aging, mediation analysis

\section{Introduction}

Patients with chronic obstructive pulmonary disease (COPD) demonstrate widely variable exercise capacities. ${ }^{1}$ A large body of evidence suggests that forced expiratory volume in 1 second $\left(\mathrm{FEV}_{1}\right)$, as an index of the mechanical changes by which $\mathrm{COPD}$ affects exercise tolerance, is poorly correlated with exercise performance, and numerous abnormalities found outside the lung such as systemic inflammation, hypoxic 
neuroendocrine activation, chronic wasting, and skeletal muscle dysfunction also impact exercise performance. ${ }^{2-4}$

Recent attention has been given to the contribution of systemic inflammation, as reflected by increased plasma levels of protein interleukin-6 (IL-6) and C-reactive protein (CRP), to reduced muscle strength, decreased exercise endurance, shorter 6-minute walk distance (6MWD), and poor health status. ${ }^{5-8}$ Protein IL-6, besides its central role in initiating and modulating acute-phase inflammatory responses to injuries and infections, increases in plasma during stress unrelated to inflammation. In particular, previous in vivo $^{9}$ and recent in vitro ${ }^{10}$ studies have suggested that oxidative stress in hard contractive respiratory muscles elicits expression of IL-6, interleukin- $1 \beta$, and tumor necrosis factor- $\alpha$, and this cytokine interplay accounts for most of the hypothalamic-pituitaryadrenal axis stimulating activity in plasma. Importantly, these hormone-like effects speak also to the possibility that cytokines constitute a separate system of regulation and defense reaction closely connected to nervous, endocrine, and metabolic regulation systems. ${ }^{11}$

Based on this literature, one can pose a research hypothesis of a mediating role for systemic cytokines on the effect of $\mathrm{FEV}_{1}$ on walking performance for individuals suffering from COPD. If this relationship can be found, it would suggest how a systemic inflammatory response impacts walking performance in COPD. In this context, the current authors hypothesized a mediation model to test whether the relationship between patients' post-bronchodilator $\mathrm{FEV}_{1}$ (independent variable) and 6MWD (outcome variable) could be at least partially explained by circulating levels of protein IL-6 (mediator or process variable). We also explored whether or not this proposed mediation model was moderated by several status variables, namely, age, sex, and body mass index (BMI).

\section{Methods}

All patients involved in the screening of the randomized controlled clinical trial of nonpharmacologic treatment (ClinicalTrials.gov identifier: NCT01253941) were recruited into the present study. Patients who had been referred to the pulmonary rehabilitation unit of Salvatore Maugeri Foundation IRCCS (Montescano, Italy) from March 2010 to March 2012 underwent a clinical/functional screening during the intake phase of the protocol. The screening encompassed presence of symptoms (Modified Medical Research Council [MMRC] Dyspnea Scale; [see BODE reference below for details]), quality of life (EuroQoL valuation questionnaire EQ-5D and visual analog scale EQ-VAS) ${ }_{1}^{12}$ pulmonary function tests, BODE (BMI, airway obstruction, dyspnea, and exercise capacity) score $0-10,{ }^{13}$ and blood biomarkers (CRP and protein IL-6). Chest radiography and thorax computed tomography scan were optionally analyzed. This reappraisal process was implemented in patients undergoing pulmonary rehabilitation before they were moved to eligibility screening and the run-in phase of the randomized clinical trial, and many individuals faced the problem of dealing with a presumptive COPD diagnosis that may not have been systematically checked in primary clinical care. All of the approached patients gave the researchers written permission to obtain sociodemographic and clinical data from their medical files. Patients who participated were requested to complete the EuroQoL questionnaire (EQ-5D and EQ-VAS) before the start of the rehabilitation program. Patients who had had primary lung conditions other than COPD were excluded from the analysis. All of the patients had to meet the American Thoracic Society (ATS) criteria for diagnosis of COPD. ${ }^{14} \mathrm{At}$ the time of examination, patients were receiving a standard treatment regimen consisting of inhaled corticosteroids and long-acting beta-adrenergic agonists, and/or long-acting anticholinergic drugs. The presence of physician-diagnosed comorbidities such as arrhythmias, cardiovascular disease, coronary artery disease, chronic pulmonary arterial hypertension, previous neoplasias, and obstructive sleep apnea syndrome were recorded. In addition, objectively identified comorbidities ${ }^{15}$ were assessed as well, namely: systemic arterial hypertension (systolic blood pressure $>140 \mathrm{mmHg}$, or diastolic blood pressure $>90 \mathrm{mmHg}$ ); hyperglycemia (fasting glucose levels $>5.6 \mathrm{mmol} / \mathrm{L}$ ); dyslipidemia (triglyceride blood levels $>1.7 \mathrm{mmol} / \mathrm{L}$ or high-density lipoprotein cholesterol level $<1.03 \mathrm{mmol} / \mathrm{L}$ in men, and $<1.26 \mathrm{mmol} / \mathrm{L}$ in women); obesity (BMI $>30 \mathrm{~kg} / \mathrm{m}^{2}$ ); underweight (BMI $<21 \mathrm{~kg} / \mathrm{m}^{2}$ ); anemia (hemoglobin level $<8.1 \mathrm{mmol} / \mathrm{L}$ in men, and $<7.5 \mathrm{mmol} / \mathrm{L}$ in women); renal impairment (estimated glomerular filtration rate $<60 \mathrm{~mL} / \mathrm{min}$ ); osteoporosis (T-score $<-2.5$ ); chronic respiratory failure (arterial oxygen tension $\left[\mathrm{PaO}_{2}\right]<8.0 \mathrm{kPa}$, and arterial carbon dioxide tension $\left[\mathrm{PaCO}_{2}\right]>6.0 \mathrm{kPa}$ ); and symptoms of anxiety and depression (EQ-5D questionnaire item 5 score $=3$ ). It was also noted whether patients were currently smoking, on long-term oxygen therapy, or taking medications or systemic corticosteroids. The medical ethics committee at our home institution approved the study design.

\section{Measurements}

Spirometry was performed using a Jaeger spirometer and body box (Masterscreen ${ }^{\circledR}$ Body; VIASYS Healthcare GmbH, 
Hoechberg, Germany). $\mathrm{FEV}_{1}$ and forced vital capacity were obtained. $\mathrm{FEV}_{1}$ was also measured 15 minutes after four inhalations of salbutamol $(400 \mu \mathrm{g})$ from a metered-dose inhaler. Predicted equations utilized were those of Quanjer et al. ${ }^{16}$

The 6-minute walking test is systematically performed at the beginning of the rehabilitation program at our institution, organized following ATS/European Respiratory Society guidelines ${ }^{17,18}$ for pulmonary rehabilitation, and consists of general physical training with particular attention to exercise in relation to daily activities. ${ }^{18}$ The $6 \mathrm{MWD}$, ie, distance walked during a standardized 6-minute walking test, is taken as an outcome measure of exercise capacity.

The 6-minute walking test was conducted as described by Guyatt et al, ${ }^{19}$ which was a modification of the 12-minute walking test originally described by McGavin et al. ${ }^{20}$ A 100-foot (30.5 m) hospital corridor course was used and was marked by colored tape at each end. Patients underwent two 6-minute walking tests at least 30 minutes apart. This procedure was used to eliminate any potential learning effect, and the second of the two walk distances was recorded. ${ }^{18}$

Performance status of the patients was assessed by BMI, degree of airflow obstruction and dyspnea, and exercise capacity (BODE index). ${ }^{13}$ These measurements constitute a multidimensional grading system based on: 1) the measurement of BMI $\left(\mathrm{kg} / \mathrm{m}^{2}\right)$; 2) $\mathrm{FEV}_{1}, \%$ predicted; 3) degree of dyspnea, measured with the MMRC Dyspnea Scale; and 4) the evaluation of exercise tolerance, as reflected by the distance walked in the 6-minute walking test (6MWD).

The EuroQoL questionnaire EQ-5D and EQ-VAS ${ }^{12}$ was administered to all of the participants; it is designed to collect evaluations for health-related quality of life states consisting of five items (mobility, self-care, usual activities, pain/discomfort, and anxiety/depression). Patients were asked to refer to their dyspnea when answering pain/discomfort items. Each item has three levels of response: no problems (1), some problems (2), and extreme problems (3). Higher scores collectively indicate more severe problems. In addition, patients rated their current health using a visual analog scale (VAS). VAS scores vary from 0 (death or worst possible health) to 100 (best possible health).

Determinations of fasting plasma levels of protein IL-6 and CRP were implemented in routine examinations performed at the start of the respiratory rehabilitation program at our institution. Fasting ethylenediaminetetraacetic acid (EDTA) venous blood samples were collected early in the morning (8-10 am). CRP was measured in duplicate by high-sensitivity particle enhanced turbidimetric immunoassay (PETIA) (Siemens AG, Munich, Germany), with a lower detection limit of $0.5 \mathrm{mg} / \mathrm{L}$.
Protein IL-6 was measured by an enzyme-linked immunosorbent assay (ELISA) kit utilizing commercial ELISA (R\&D Systems, Inc., Minneapolis, MN, USA).

Arterial blood samples were gently drawn from the brachial artery, using a dedicated preheparinized blood sampler, while patients were seated and breathing room air. Oxygen breathing was temporarily withdrawn for at least 30 minutes in patients undergoing oxygen therapy. Immediately after sample collection, mixing, and removal of the first drops of blood, the acid-base and oxygen status indices were analyzed. Arterial negative logarithm of hydrogen ion concentration $(\mathrm{pH})$, $\mathrm{PaCO}_{2}, \mathrm{PaO}_{2}$, and the oximetry parameters were measured using a blood gas analyzer (ABL 700 system; Radiometer Medical ApS, Brønshøj, Denmark).

\section{Mediation analyses}

To test our mediation hypothesis, we proposed a temporal chain in which the intervening variable (IL-6) functioned as the mediator of the effect of $\mathrm{FEV}_{1}$ on $6 \mathrm{MWD}$ performance. Specifically, it was predicted that $\mathrm{FEV}_{1}$ (X variable) would negatively predict IL-6 levels (M variable), and that IL-6, in turn, would negatively predict the outcome of 6MWD (Y variable). A three-step statistical approach was used to obtain estimates of mediation effects. In step 1, a regression analysis with $\mathrm{X}$ predicting $\mathrm{Y}$ alone was conducted to estimate path $\mathrm{c}$ (total effect). In step 2, the relationship of $\mathrm{X}$ predicting $\mathrm{M}$ was conducted to estimate path a. In step 3, a regression was performed with $\mathrm{X}$ and $\mathrm{M}$, simultaneously predicting $\mathrm{Y}$ to estimate the size of path $\mathrm{b}$. The indirect effect of mediation, in which $\mathrm{X}$ leads to $\mathrm{Y}$ through $\mathrm{M}$, is assessed by estimating the product of $\mathrm{a} \times \mathrm{b}$, and it represents the portion of the original relationship between $\mathrm{X}$ and $\mathrm{Y}$ that is mediated by $\mathrm{M}$, while the direct effect (path c') is the residual effect of $\mathrm{X}$ on $\mathrm{Y}$ controlling for $\mathrm{M}$. To test statistical significance of the mediation pattern, a bootstrapped mediation test was performed in SPSS Amos software (v 19; IBM Corporation, Armonk, NY, USA) with 4,000 resamplings and a 95\% confidence interval (CI). Computation of the size of the mediation effect was based on standardized regression coefficients according to methods described by Jose ${ }^{21}$ and MacKinnon. ${ }^{22}$ Further analyses were performed to ascertain whether status variables might function as moderators of this obtained mediation result. Three moderators were examined: sex, age, and BMI status.

\section{Statistical analyses}

Reports of data are expressed in this report as mean and standard deviation, unless otherwise stated. Descriptive statistics 
of both protein IL-6 and CRP showed that these variables manifested non-normal distributions (Kolmogorov-Smirnov $\mathrm{D}=0.42, P<0.001$ and $\mathrm{D}=0.31, P<0.001$, respectively). These variables were transformed into log units.

As past research has shown that risk and mortality for cardiovascular disease increases with IL-6 levels, with the most pronounced risk at the uppermost quartile, ${ }^{23}$ we also measured IL-6 plasma levels in 16 healthy subjects (isolated control group), and used the upper quartile value of IL-6 distribution as the cut point for defining high levels of this protein.

Zero-order correlations of parametric data were performed by the Pearson method. Differences of proportions were tested by Pearson $\chi^{2}$. A $P$-value $<0.05$ was considered to be statistically significant.

\section{Results}

\section{Descriptive statistics}

Overall, 180 patients were approached and recruited. The appropriateness of COPD diagnosis was appraised in 153 individuals. A COPD diagnosis was excluded in 27 patients. Characteristics of the 153 patients (123 males) in total and within each Global initiative for chronic Obstructive Lung Disease (GOLD) functional stage ${ }^{14}$ are shown in Table 1 . Out of 153 individuals, 71 patients $(46 \%)$ fell within GOLD stage III or IV.

Most of the patients were ex-smokers (73\%) or current smokers (17\%), with a median number of smoked pack-years of 45 (interquartile range [IQR] 42-179.3).
Figure 1 shows comorbidity frequencies ranging from $4 \%$ to $75 \%$. Dyslipidemia, systemic arterial hypertension, hyperglycemia, and chronic respiratory failure were the four most prevalent comorbidities. Almost all patients had one or more comorbidity with a median of 5 (IQR 3-8). Among COPD patients segregated by median age, the ratio of males to females increased with age: 53 males to 19 females in the group younger than 70 years, and 70 males to eleven females in the group over 70 years $\left(\chi^{2}=3.97, P=0.046\right)$. The ratio of individuals manifesting high versus low levels of IL-6 (cutoff value $=5.4 \mathrm{pg} / \mathrm{mL}$ ) was 38:34 in the younger group and 56:25 in the older group $\left(\chi^{2}=4.31, P=0.038\right)$. The self-care item of the EQ-5D questionnaire showed that more problems were reported with increasing age $\left(\chi^{2}=7.19, P=0.028\right)$. On the other hand, health status (VAS score $\leq 50$ ) did not significantly vary by age $(P=0.47)$.

A statistically significant correlation between CRP and cytokine IL-6 $\left(R^{2}=0.65,95 \% \mathrm{CI}: 0.55-0.73 ; P<0.001\right)$ was found, indicating a strong association between the two variables.

The majority of patients (73\%) were on inhaled corticosteroid therapy. Out of the overall sample of 153 , 33 patients $(22 \%)$ were scaling down systemic steroids at the time of the study; $58 \%$ of the patients were on antihypertensive drugs; $10 \%$ of patients were on statins; $42.5 \%$ of patients were on long-term oxygen therapy; $25 \%$ were on noninvasive continuous positive pressure

Table I Descriptive statistics of I53 patients (I23 males) with COPD, overall and in each functional GOLD stage

\begin{tabular}{|c|c|c|c|c|c|}
\hline & $\begin{array}{l}\text { Total } \\
n=153\end{array}$ & $\begin{array}{l}\text { GOLD I } \\
n=54\end{array}$ & $\begin{array}{l}\text { GOLD II } \\
\mathbf{n}=\mathbf{2 8}\end{array}$ & $\begin{array}{l}\text { GOLD III } \\
n=34\end{array}$ & $\begin{array}{l}\text { GOLD IV } \\
n=37\end{array}$ \\
\hline Males, $\mathrm{n}$ & 123.0 & 43.0 & 21.0 & 27.0 & 32 \\
\hline Age, years & $70.1(9.0)$ & $67.0(11.4)$ & $72.2(7.6)$ & $71.6(8.6)$ & $71.5(7.3)$ \\
\hline \multicolumn{6}{|l|}{ Smoking habit } \\
\hline CS, n & 22 & 10 & 4 & 3 & 4 \\
\hline $\mathrm{FS}, \mathrm{n}$ & 96 & 26 & 16 & 28 & 26 \\
\hline NS, n & 13 & 7 & 4 & 5 & - \\
\hline \multicolumn{6}{|l|}{ BODE index } \\
\hline BMI, kg/m² & 27.7 (6.9) & 30.1 (6.5) & $28.2(8.1)$ & $25.3(5.8)$ & $24 . I(6 . I)$ \\
\hline $\mathrm{FEV}_{1}, \%$ predicted & $53.4(2 \mid .2)$ & $79.7(17.6)$ & $55.8(4.3)$ & $42.1(5.5)$ & $27.7(5.7)$ \\
\hline MMRC-D, points & $2.7(0.9)$ & $2.1(0.6)$ & $2.6(1.0)$ & $2.9(0.7)$ & $3.4(0.8)$ \\
\hline 6MWD, meters & $294.1(134.1)$ & $373.0(125.1)$ & $278.9(111.5)$ & $259.6(109.8)$ & $217.2(117.1)$ \\
\hline BODE points & $4.3(2.8)$ & $\mathrm{I} .7(\mathrm{I} .4)$ & $3.9(1.6)$ & $5.6(I .8)$ & $7.5(2.0)$ \\
\hline \multicolumn{6}{|l|}{ Pulmonary function } \\
\hline $\mathrm{FEV}_{\mathrm{I}} / \mathrm{FVC}, \%$ & $56.3(17.5)$ & $66.1(9.5)$ & $61.2(9.0)$ & $46.1(15.5)$ & $35.6(8.9)$ \\
\hline $\mathrm{PaCO}_{2}, \mathrm{kPa}$ & $5.3(I . I)$ & $5.1(0.8)$ & $5.1(0.9)$ & $5.6(1.2)$ & $5.9(1.4)$ \\
\hline $\mathrm{PaO}_{2}, \mathrm{kPa}$ & $8.8(1.4)$ & $9.4(1.5)$ & 9.1 (I.2) & $8.5(1.0)$ & $7.8(I . I)$ \\
\hline $\mathrm{P}_{50}, \mathrm{kPa}$ & $3.3(0.3)$ & $3.2(0.3)$ & $3.2(0.3)$ & $3.3(0.4)$ & $3.3(0.3)$ \\
\hline
\end{tabular}

Note: Data are presented as mean (standard deviation) unless stated otherwise.

Abbreviations: COPD, chronic obstructive pulmonary disease; GOLD, Global initiative for chronic Obstructive Lung Disease; CS, current smokers; FS, former smokers; NS, nonsmokers; BODE, BMI, degree of airflow obstruction, dyspnea, and exercise capacity; BMI, body mass index; FEV ${ }_{1}$, forced expiratory volume in I second; MMRC-D, Modified Medical Research Council Dyspnea Scale; 6MWD, 6-minute walk distance; FVC, forced vital capacity; $\mathrm{PaCO}_{2}$, arterial carbon dioxide tension; PaO ${ }_{2}$, arterial oxygen tension; $P_{50}$, oxygen half-saturation tension. 


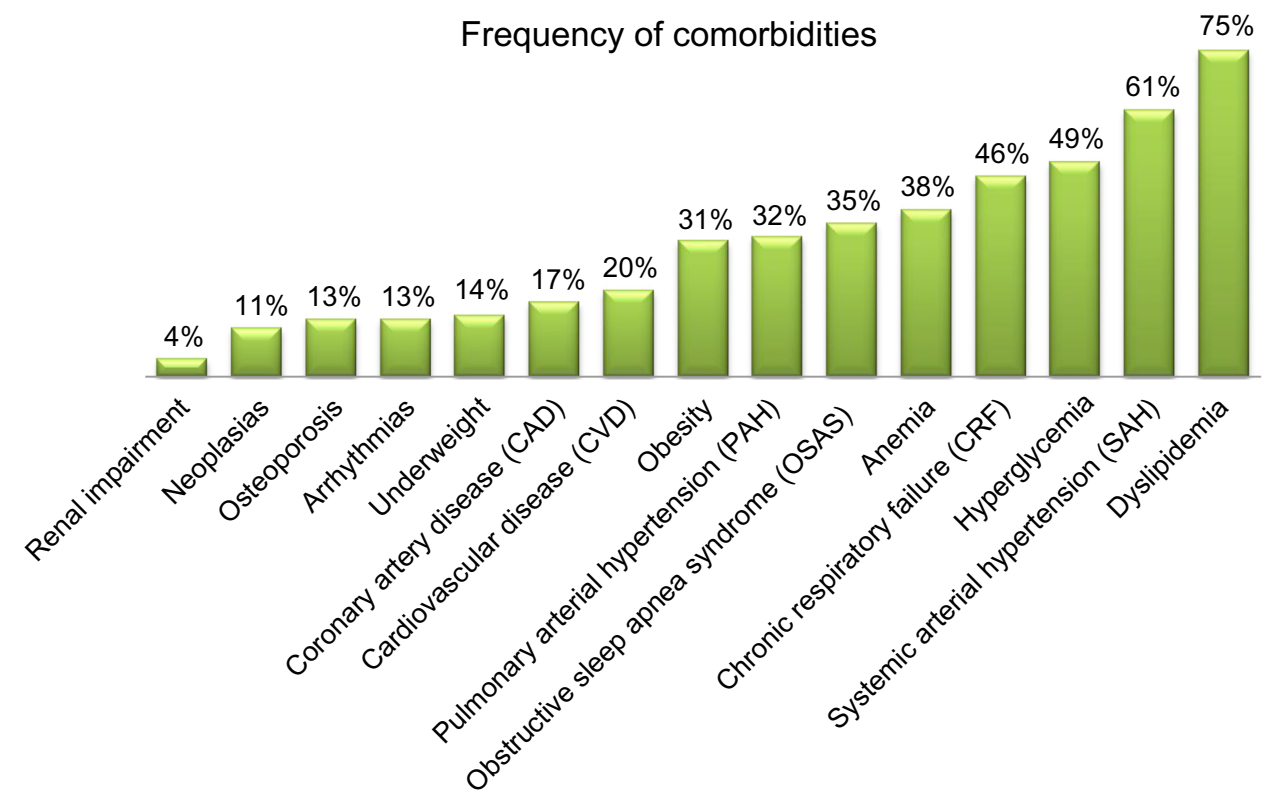

Figure I Frequency of comorbidities in I53 patients suffering from chronic obstructive pulmonary disease.

ventilation; and $10 \%$ were on bilevel noninvasive positive pressure ventilation.

Table 2 shows anthropometrics, spirometry, IL-6, and CRP descriptive statistics of 16 healthy subjects (isolated control group). Out of 153 COPD patients, 59 (38.6\%) manifested protein IL-6 plasma levels below, and 94 (61.4\%) above, $5.4 \mathrm{pg} / \mathrm{mL}$, which was the upper quartile of the distribution produced by the isolated control group.

Table 3 shows the zero-order correlations between FEV and 6MWD, between FEV 1 and IL-6 in log units (lnIL-6), and between InIL-6 and 6MWD. These relationships confirmed the expectation that $\mathrm{FEV}_{1}$ and $6 \mathrm{MWD}$ would be positively and moderately related, and, as expected, lnIL-6 was negatively related to both variables. Since size and statistical significance of these relationships suggested that a significant mediation

Table 2 Descriptive statistics in 16 healthy controls (six males)

\begin{tabular}{lllll}
\hline Subjects & $\begin{array}{l}\text { Mean/ } \\
\text { [median] }\end{array}$ & 95\% Cl/[IQR] & Ist Q & 3rd Q \\
\hline Age, years & 47.9 & $45.2-50.6$ & 42.8 & 51.8 \\
BMI, kg/m² & 25.9 & $23.9-27.9$ & 23.8 & 28.5 \\
FEV $_{1}, \%$ predicted & 108.9 & $101.4-116.4$ & 99.0 & 121.5 \\
FEV $/$ /FVC, \% & 80.2 & $77.8-82.6$ & 76.9 & 82.8 \\
IL-6, Pg/mL & {$[4.6]$} & {$[2.1-4.9]$} & 3.3 & 5.4 \\
CRP, mg/L & {$[1.3]$} & {$[1.1-3.2]$} & 1.1 & 2.2 \\
\hline
\end{tabular}

Note: Data are presented as mean or [median] in the first column, $95 \% \mathrm{Cl}$, or median and QR.

Abbreviations: $\mathrm{Cl}$, confidence interval; IQR, interquartile range; Ist $\mathrm{Q}$, first quartile; 3rd Q, third quartile; BMI, body mass index; $\mathrm{FEV}_{1}$, forced expiratory volume in I second; FVC, forced vital capacity; IL-6, cytokine interleukin 6; CRP, C-reactive protein. might be present, we computed a bootstrapped mediation model in the AMOS software to test this hypothesis.

\section{Mediation analysis}

Figure 2 depicts the mediation path model. The numerical values associated with the three pathways are standardized regression weights (or betas). The reduction of the raw correlation between $\mathrm{FEV}_{1}$ and $6 \mathrm{MWD}$ (total effect) from 0.52 to 0.43 with inclusion of the mediator is indicative of a significant mediational result, but the size of the decrease has to be determined. Bootstrapping test output obtained evidence of a statistically significant mediation result: standardized indirect effect $=0.089$, standard error $=0.03,95 \%$ CI: $0.041-0.164$, $P=0.003$. Computation of the size of the mediation effect based on standardized regression coefficients yielded the

Table 3 Raw correlations between FEV,\% of predicted, InIL-6, and 6MWD

\begin{tabular}{lll}
\hline & InIL-6 & 6MWD \\
\hline FEV $\%$ predicted & & \\
Pearson correlation & $-0.268^{* *}$ & $0.522^{* *}$ \\
Significance (two-tailed) & $<0.001$ & $<0.000$ \\
N & 153 & 153 \\
InIL-6 & & \\
Pearson correlation & & $-0.447^{* *}$ \\
Significance (two-tailed) & & 0.000 \\
N & & 153 \\
\hline
\end{tabular}

Note: $* * P$-value $<0.01$.

Abbreviations: $\mathrm{FEV}_{1}$, forced expiratory volume in I second; InL-6, log of cytokine interleukin 6 plasma levels; 6MWD, 6-minute walk distance. 


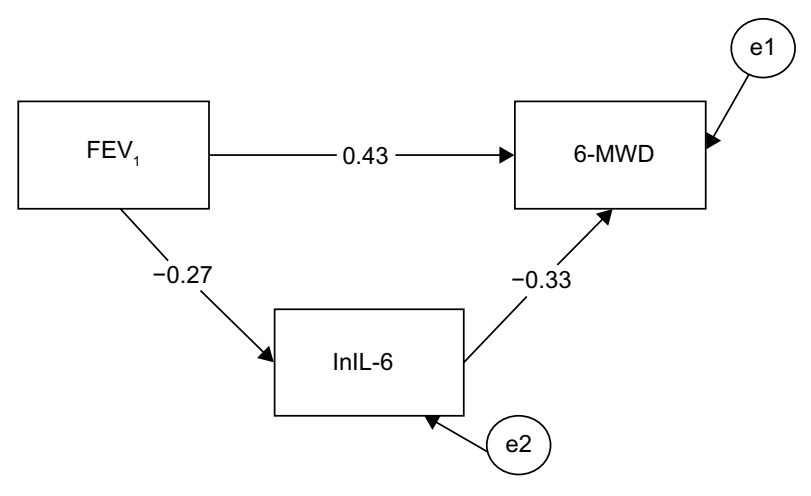

Figure 2 Mediation path model for the entire sample $(\mathrm{N}=153)$.

Notes: Inclusion of the mediator reduces the row correlation coefficient between FEV, and 6MWD (total effect $=0.52$ ). The numbers are the correlation coefficients of the mediation paths: path $c^{\prime}=0.43$, path $a=-0.27$, path $b=-0.33$. Circles with the letter " $\mathrm{e}$ " refer to error or residual.

Abbreviations: 6MWD, 6-minute walk distance; $\mathrm{FEV}_{1}$, forced expiratory volume in I second; InIL-6, log of cytokine interleukin 6 levels. information that the mediational path involving lnIL-6 accounted for $17 \%$ of the total effect. This result suggests that increase of airflow obstruction (a lowering of $\mathrm{FEV}_{1}$ ) predicted an increase in lnIL-6 levels, which, in turn, predicted a decrease in walking performance (6MWD).

Among the status variables tested as moderators of this obtained result, neither sex nor BMI evidenced any significant influence on the obtained mediational pattern, but age was found to operate as a significant moderator.

Figure 3 shows that age moderated the obtained overall mediation pattern. In particular, after splitting the sample by median age ( 70 years), we obtained a significant mediation result for individuals younger than 70 years: standardized indirect effect $=0.122$, se $=0.05,95 \%$ CI: $0.044-0.254, P=0.004$. In contrast, a nonsignificant mediation result was obtained for
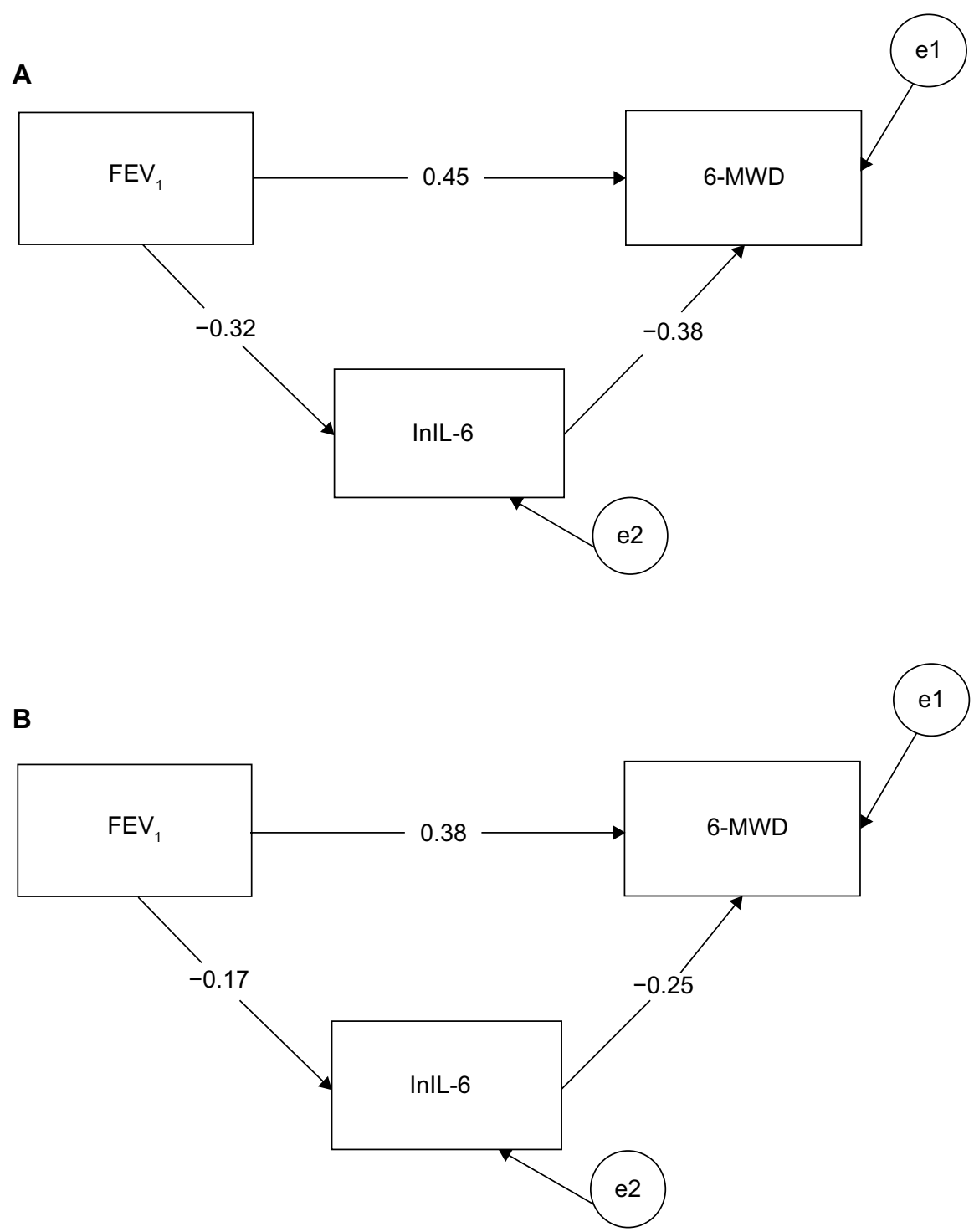

Figure 3 Mediation path models for participants according to age.

Notes: The numbers are the correlation coefficients of the mediation paths: path c', path a, and respectively path b. (A) The mediation path model for participants younger than 70 years $(\mathrm{N}=69)$. (B) The mediation path model for participants older than 70 years $(\mathrm{N}=84)$. Circles with the letter " $\mathrm{e}$ " refer to error or residual.

Abbreviations: 6MWD, 6-minute walk distance; FEV , forced expiratory volume in I second; InlL-6, log of cytokine interleukin 6 plasma levels. 
individuals older than 70 years: standardized indirect effect $=0.044$, se $=0.03,95 \%$ CI: -0.010 to $0.142, P>0.10$. The "a" path (from $X$ to $M$ variable) and the "b" path (from $M$ to $Y$ variable) were not significantly different in strength between the younger and older groups; however, the younger group manifested stronger betas (standardized regression coefficients) for both the a ( $\beta$ : -0.32 versus -0.17$)$ and $b$ paths $(\beta:-0.38$ versus -0.25 ) compared to the older group. Since the strength of the indirect effect is computed by multiplying path a by path $b$, it is clear that the size of the indirect effect in relation to the total effect was larger for younger individuals $(0.21)$ than for the older individuals (0.10). This result suggests that lnIL-6 levels mediated between severity of airflow obstruction and walking performance among elderly adults to about half the extent they did among younger adults.

\section{Discussion}

The twin findings of the current study, namely: 1) the mediation of cytokine IL-6 circulating levels on the effect of FEV upon walking performance in patients with COPD and 2) the moderation of this effect by age, are consistent with suggestions that protein IL-6 is one of the main signaling pathways modulating the complex relationship between aging and chronic morbidity. ${ }^{24}$

The novelty of the present study stems from our choice to analyze the cross-sectional observations obtained in a sample of older individuals with COPD by using a mediation model to investigate the proposed sequence of severity of airflow obstruction $\rightarrow$ increasing IL-6 blood levels $\rightarrow$ impairment of 6MWD, in order to better understand how living with COPD impacts walking performance. Moreover, the potential moderating influence of several status variables on the mediated effect was tested, with the finding that neither BMI nor sex moderated its strength, but age did. The present statistical approach has been borrowed from psychological theory and research, wherein the mediation-moderation framework is very popular. ${ }^{25}$ The logic of a mediation analysis, namely, assessing the dynamic interplay of several related variables, seems to apply well to the phenomenon of protein IL-6 circulating in blood, based on the rationale that its biological role is to transmit immune, metabolic, neural, and hormonal signaling along specific pathways.

Protein IL-6 plays different biological roles in the context of the acute phase response, as well as in the progression from acute to chronic inflammation. In acute airway and alveolar inflammation, the role of protein IL-6 has been documented by studies showing its association with faster decline in lung function, ${ }^{26}$ and it is related to exacerbations in patients with COPD. ${ }^{27}$ In the pathogenesis of chronic inflammation, IL-6 trans-signaling presumably sustains the switch from the inflammatory burst that follows an inflammatory stimulus to chronic elevation of IL- $6 .{ }^{28}$ In addition, the stress-related neural, endocrine, and metabolic actions of the triad of tumor necrosis factor- $\alpha$, interleukin-1, and IL-6 are relevant to pulmonary rehabilitation interventions, in that these systemic mediators intervene in an adaptive response elicited to prevent fatigue or reduce injury to hard contractive respiratory muscles ${ }^{9,10}$ and play a part in the metabolic changes associated with chronic wasting of COPD and in its adverse effect on physical performance. ${ }^{29}$ In particular, the well-established IL-6 release, acting as an energetic sensor of reduced fuel stores to exercising muscles, ${ }^{30}$ needs to be taken into account.

Along this line of reasoning, the finding that BMI does not moderate the mediating role of IL-6 on 6MWD implies that low-grade systemic inflammation operates similarly in weight-losing, normal weight, and obese (non-weight losing) patients; and leads to a more or less manifest fat-free mass depletion and COPD sarcopenia, as previously reported by Eid et al. ${ }^{31}$ Further, the strong correlation between CRP and IL-6 blood levels found here provides circumstantial evidence for the inference we made in a previous pilot study ${ }^{32}$ concerning treating an increased CRP blood level as a marker of IL-6 overproduction in response to impaired signaling to glycogen synthesis in weight-losing patients with COPD undergoing nutritional supplementation. Therefore, we are motivated to suggest from our mediation result that protein IL-6 mediates the effect of airflow obstruction on development of a frailty status (as reflected by a reduced 6MWD), in agreement with previous findings in geriatric population samples. ${ }^{33,34}$

The finding that the indirect effect of IL-6 on 6MWD diminishes in elderly COPD patients further reinforces the basis for speculating that cytokines also function as a defense reaction closely connected to the neuroendocrine dysregulation of aging, as previously reported in other research investigating the interrelations between protein IL-6 overproduction in response to changes relevant to dehydroepiandrosterone and cortisol circulating levels in aging males and females. ${ }^{35,36}$ Alternatively, it is tempting to interpret the moderating effect of age on the mediation effect identified here to be the result of a modulation of key features of COPD by cell senescence, resulting in enhancement of inflammatory phenotype by cytokines released by senescent cells, paracrine loops of cytokine secretion, and further activation of senescent cells. ${ }^{37}$

Overall, we would argue that the results of the moderated mediation analysis in this study lend support to the idea of a contribution of lung aging to COPD progression, and of aging intertwining with several age-related comorbidities via 
activation of a systemic inflammatory response and a intrapulmonary adaptive immune response, as well as a neuroendocrine and metabolic dysregulation that have been previously identified, but which are not yet fully understood. ${ }^{38,39}$

In the field of pulmonary rehabilitation, the results of the moderated mediation analysis call for implementation of specific intervention studies aimed at evaluating whether or not general physical training impacts the temporal sequence of events and the covariation patterns among variables identified by using the present concurrent dataset.

We acknowledge that our study has several limitations. First, we acknowledge that measurement of IL-6 blood levels does not necessarily reflect the levels of biologically active protein IL-6 in a person's bloodstream. IL-6 biological activity is dependent on binding with cognate IL-6 receptor (CD126) in cell types expressing it, and also is affected by trans-signaling activity of the complex formed by IL-6 with soluble IL-6 receptor (sIL-6R) after proteolytic shedding from the surface of neutrophils and monocytes, or modulated from inactivation of the circulating IL-6/sIL-6R receptor complex. $^{25,40,41}$ Second, we are aware that a base determination of IL-6 obtained at one point in time can provide only limited information on the general or typical degree of activation of the IL-6 pathway. Nevertheless, CRP is acknowledged as one of the powerful stimulators of the shedding process. ${ }^{42,43}$ Thus, the close relationship between IL-6 and CRP suggests the possibility for a systematic activation and amplification of IL-6 signaling by the shedding process. It is also acknowledged that results of mediation analyses are most illuminating when they are based on longitudinal data; since we used concurrent data in the present study, we are precluded from making a causal argument among the three main variables studied here. Nevertheless, mediation analyses with concurrent datasets are useful for identifying patterns of covariation among variables that stand as promising candidates for further research with longitudinal datasets. We suggest that the identified mediational pattern obtained in the present data should be evaluated with longitudinal data. Finally, the overrepresentation of males compared to females in our sample of COPD patients, with a 4:1 male-to-female ratio, should be regarded as a potential limitation in that it might have hampered evaluation of sex as a moderator of our mediational model.

\section{Conclusion}

The main result of the present study, that systemic immune activation partially mediates the impact of $\mathrm{FEV}_{1}$, the classical pulmonary benchmark, on walking performance decline, is suggestive of a dynamic relationship, although it cannot authoritatively demonstrate a cause and effect relationship. Thus, longitudinal or intervention studies are needed to further elucidate how functional impairment leads to frailty status and disability in advanced COPD.

\section{Acknowledgments}

The authors acknowledge Dr Paola Abelli and Dr Rosanna Catella, medical directors at the Scientific Institute of Montescano. The authors would also like to thank the patients who participated in the study.

\section{Author contributions}

Study idea and design: SB, PEJ, and CB. Statistical analysis: PEJ. Data acquisition: ED, PC, and SM. Interpretation of results: SB, CB, AR, GDP, RM, EB, and CF. Manuscript drafting/revision: SB and PEJ. All of the authors have read the manuscript and agree with the content contained within. All authors contributed toward data analysis, drafting and revising the paper and agree to be accountable for all aspects of the work.

\section{Disclosure}

The authors report no conflicts of interest in this work.

\section{References}

1. Bauerle $\mathrm{O}$, Younes M. Role of ventilatory response to exercise in determining exercise capacity in COPD. J Appl Physiol (1985). 1995;79(6): 1870-1877.

2. Croxton TL, Weinmann GG, Senior RM, Wise RA, Crapo JD, Buist AS. Clinical research in chronic obstructive pulmonary disease: needs and opportunities. Am J Respir Crit Care Med. 2003;167(8): $1142-1149$

3. Reid MB. COPD as a muscle disease. Am J Respir Crit Care Med. 2001;164(7):1101-1102.

4. Wouters EF. Chronic obstructive pulmonary disease. 5: systemic effects of COPD. Thorax. 2002;57(12):1067-1070.

5. Agustí AG, Noguera A, Sauleda J, Sala E, Pons J, Busquets X. Systemic effects of chronic obstructive pulmonary disease. Eur Respir J. 2003;21(2):347-360.

6. Kawakami Y, Kishi F, Yamamoto H, Miyamoto K. Relation of oxygen delivery, mixed venous oxygenation, and pulmonary hemodynamics to prognosis in chronic obstructive pulmonary disease. N Engl J Med. 1983;308(18):1045-1049.

7. Broekhuizen R, Wouters EF, Creutzberg EC, Schols AM. Raised CRP levels mark metabolic and functional impairment in advanced COPD. Thorax. 2006;61(1):17-22.

8. Pinto-Plata VM, Cote C, Cabral H, Taylor J, Celli BR. The 6-min walk distance: change over time and value as a predictor of survival in severe COPD. Eur Respir J. 2004;23(1):28-33.

9. Vassilakopoulos T, Zakynthinos S, Roussos C. Strenuous resistive breathing induces proinflammatory cytokines and stimulates the HPA axis in humans. Am J Physiol. 1999;277(4 Pt 2): R1013-R1019.

10. Sigala I, Zacharatos $P$, Boulia $S$, et al. Nitric oxide regulates cytokine induction in the diaphragm in response to inspiratory resistive breathing. J Appl Physiol (1985). 2012;113(10):1954-1603.

11. Aleksandrova NP. [Cytokines and resistive breathing]. Fiziol Cheloveka. 2012;38(2):119-129. Russian. 
12. EuroQol Group. EuroQol - a new facility for the measurement of health-related quality of life. Health Policy. 1990;16(3):199-208.

13. Celli BR, Cote CG, Marin JM, et al. The body-mass index, airflow obstruction, dyspnea, and exercise capacity index in chronic obstructive pulmonary disease. $N$ Engl J Med. 2004;350(10):1005-1012.

14. Vestbo J, Hurd SS, Agustí AG, et al. Global strategy for the diagnosis, management, and prevention of chronic obstructive pulmonary disease: GOLD executive summary. Am J Respir Crit Care Med. 2013;187(4): 347-365.

15. Vanfleteren LE, Spruit MA, Groenen M, et al. Clusters of comorbidities based on validated objective measurements and systemic inflammation in patients with chronic obstructive pulmonary disease. Am J Respir Crit Care Med. 2013;187(7):728-735.

16. Quanjer PH, Tammeling GJ, Cotes JE, Pedersen OF, Peslin R, Yernault JC. Lung volumes and forced ventilatory flows. Report Working Party Standardization of Lung Function Tests, European Community for Steel and Coal. Official Statement of the European Respiratory Society. Eur Respir J Suppl. 1993;16:5-40.

17. Spruit MA, Singh SJ, Garvey C, et al. ATS/ERS Task Force on Pulmonary Rehabilitation. An official American Thoracic Society/ European Respiratory Society statement: key concepts and advances in pulmonary rehabilitation. Am J Respir Crit Care Med. 2013;188(8): e13-e64.

18. ATS Committee on Proficiency Standards for Clinical Pulmonary Function Laboratories. ATS statement: guidelines for the six-minute walk test. Am J Respir Crit Care Med. 2002;166(1):111-117.

19. Guyatt GH, Pugsley SO, Sullivan MJ, et al. Effect of encouragement on walking test performance. Thorax. 1984;39(11):818-822.

20. McGavin CR, Gupta SP, McHardy GJ. Twelve-minute walking test for assessing disability in chronic bronchitis. Br Med J. 1976;1(6013): 822-823.

21. Jose PE. Doing Statistical Mediation and Moderation. New York, NY: The Guilford Press; 2013.

22. MacKinnon DP. Introduction to Statistical Mediation Analysis. Mahway, NJ: Lawrence Erlbaum Associates; 2008.

23. Cesari M, Penninx BW, Newman AB, et al. Inflammatory markers and cardiovascular disease (The Health, Aging and Body composition [Health ABC] Study). Am J Cardiol. 2003;92(5):522-528.

24. Maggio M, Guralnik JM, Longo DL, Ferrucci L. Interleukin-6 in aging and chronic disease: a magnificent pathway. J Gerontol A Biol Sci Med Sci. 2006;61(6):575-584.

25. MacKinnon DP, Fairchild AJ, Fritz MS. Mediation analysis. Annu Rev Psychol. 2007;58:593-614.

26. Donaldson GC, Seemungal TA, Patel IS, et al. Airway and systemic inflammation and decline in lung function in patients with COPD. Chest. 2005;128(4):1995-2004.

27. Wedzicha JA, Seemungal TA, MacCallum PK, et al. Acute exacerbations of chronic obstructive pulmonary disease are accompanied by elevation of plasma fibrinogen and serum IL-6 levels. Thromb Haemost. 2000;84(2):210-215.

28. Bauer J, Bauer TM, Kalb T, et al. Regulation of interleukin 6 receptor expression in human monocytes and monocyte-derived macrophages. Comparison with the expression in human hepatocytes. J Exp Med. 1989;170(5):1537-1549.
29. Schols AM, Buurman WA, Staal van den Brekel AJ, Dentener MA, Wouters EF. Evidence for a relation between metabolic derangement and increased levels of inflammatory mediators in a subgroup of patients with chronic obstructive pulmonary disease. Thorax. 1996;51(8): 819-824.

30. Pedersen BK, Hoffman-Goetz L. Exercise and the immune system: regulation, integration, and adaptation. Physiol Rev. 2000;80(3): $1055-1081$.

31. Eid AA, Ionescu AA, Nixon LS, et al. Inflammatory response and body composition in chronic obstructive pulmonary disease. Am J Respir Crit Care Med. 2001;164(8 Pt 1):1414-1418.

32. Baldi S, Aquilani R, Pinna GD, Poggi P, De Martini A, Bruschi C. Fat-free mass change after nutritional rehabilitation in weight losing COPD: role of insulin, C-reactive protein and tissue hypoxia. Int $J$ Chron Obstruct Pulmon Dis. 2010;5:29-39.

33. Sarkar D, Fisher PB. Molecular mechanisms of aging-associated inflammation. Cancer Lett. 2006;236(1):13-23.

34. Taaffe DR, Harris TB, Ferrucci L, Rowe J, Seeman TE. Cross-sectional and prospective relationships of interleukin-6 and C-reactive protein with physical performance in elderly persons: MacArthur studies of successful aging. J Gerontol A BiolSci Med Sci. 2000;55(12):M709-M715.

35. Young DG, Skibinski G, Mason JI, James K. The influence of age and gender on serum dehydroepiandrosterone sulphate (DHEA-S), IL-6, IL-6 soluble receptor (IL-6 sR) and transforming growth factor beta 1 (TGF-beta1) levels in normal healthy blood donors. Clin Exp Immunol. 1999;117(3):476-481.

36. Straub RH, Konecna L, Hrach S, et al. Serum dehydroepiandrosterone (DHEA) and DHEA sulfate are negatively correlated with serum interleukin-6 (IL-6), and DHEA inhibits IL-6 secretion from mononuclear cells in man in vitro: possible link between endocrinosenescence and immunosenescence. J Clin Endocrinol Metab. 1998;83(6): 2012-2017.

37. Tuder RM, Kern JA, Miller YE. Senescence in chronic obstructive pulmonary disease. Proc Am Thorac Soc. 2012;9(2):62-63.

38. Freund A, Orjalo AV, Desprez PY, Campisi J. Inflammatory networks during cellular senescence: causes and consequences. Trends Mol Med. 2010;16(5):238-246.

39. Faner R, Rojas M. Macnee W, Agustí A. Abnormal lung aging in chronic obstructive pulmonary disease and idiopathic pulmonary fibrosis. Am J Respir Crit Care Med. 2012;186(4):306-313.

40. Cesari M, Penninx BW, Pahor M, et al. Inflammatory markers and physical performance in older persons: the InCHIANTI study. J Gerontol A Biol Sci Med Sci. 2004;59(3):242-248.

41. Müllberg J, Schooltink H, Stoyan T, et al. The soluble interleukin-6 receptor is generated by shedding. Eur J Immunol. 1993;23(2): 473-480.

42. Desgeorges A, Gabay C, Silacci P, et al. Concentrations and origins of soluble interleukin 6 receptor-alpha in serum and synovial fluid. J Rheumatol. 1997;24(8):1510-1516.

43. Jones SA, Novick D, Horiuchi S, Yamamoto N, Szalai AJ, Fuller GM. C-reactive protein: a physiological activator of interleukin 6 receptor shedding. J Exp Med. 1999;189(3):599-604.
International Journal of COPD

\section{Publish your work in this journal}

The International Journal of COPD is an international, peer-reviewed journal of therapeutics and pharmacology focusing on concise rapid reporting of clinical studies and reviews in COPD. Special focus is given to the pathophysiological processes underlying the disease, intervention programs, patient focused education, and self management protocols.

\section{Dovepress}

This journal is indexed on PubMed Central, MedLine and CAS. The manuscript management system is completely online and includes a very quick and fair peer-review system, which is all easy to use. Visit $\mathrm{http}: / / \mathrm{ww}$.dovepress.com/testimonials.php to read real quotes from published authors. 